\title{
PERTURBATION RESULTS FOR SOME NONLINEAR EQUATIONS INVOLVING FRACTIONAL OPERATORS
}

\author{
SIMONE SECCHI
}

Abstract. By using a perturbation technique in critical point theory, we prove the existence of solutions for two types of nonlinear equations involving fractional differential operators.

Mathematics subject classification (2010): 35Q55, 35A15, 35J20.

Keywords and phrases: Perturbation methods, pseudo-relativistic Hartree equation, fractional laplacian.

\section{REFERENCES}

[1] A. Ambrosetti, M. Badiale, And S. Cingolani, Semiclassical states of nonlinear Schrödinger equations, Arch. Rational Mech. Anal. 140, No.3 (1997), 285-300.

[2] A. Ambrosetti, A. Malchiodi, Perturbation methods and semilinear elliptic problems on $\mathbb{R}^{n}$, volume 240 of Progress in Mathematics, Birkhäuser Verlag, Basel, 2006.

[3] A. Ambrosetti, A. Malchiodi, And S. Secchi, Multiplicity results for some nonlinear Schrödinger equations with potentials, Arch. Ration. Mech. Anal. 159, No.3 (2001), 253-271.

[4] M. BERTI, P. BOLLE, Homoclinics and chaotic behavior for perturbed second order systems, Annali di Matematica Pura ed applicata, CLXXVI (1999), 323-378.

[5] L. Caffarelli, L. Silvestre, An extension problem related to the fractional Laplacian, Comm. Partial Differential Equations, 32 (2007), 1245-1260.

[6] S. Cingolani, S. SeCChi And M. SQuassina, Semi-classical limit for Schrödinger equations with magnetic field and Hartree-type nonlinearities, Proc. Roy. Soc. Edinburgh Sect. A, 140 No.5 (2010), 973-1009.

[7] V. Coti Zelati, M. Nolasco, Existence of ground states for nonlinear, pseudorelativistic Schrödinger equations, Rend. Lincei Mat. Appl., 22 (2011), 51-72.

[8] V. Coti Zelati, M. Nolasco, Ground states for pseudo-relativistic Hartree equations of critical type, Preprint 2012.

[9] E. Di Nezza, G. Palatucci and E. Valdinoci, Hitchhiker's guide to the fractional Sobolev spaces, Bull. Sci. Math., 136, No.5 (2012), 521-573.

[10] S. DipierRo, G. PALATUCCI AND E. VALDINOCI, Existence and symmetry results for a Schrödinger type problem involving the fractional laplacian, Le Matematiche, 68, n.1 (2013).

[11] P. Felmer, A. QuaAs AND J. TAN, Positive solutions of the nonlinear Schrödinger equation with the fractional laplacian, To appear on Proc. Roy. Soc. Edinburgh Sect A.

[12] R.L. FRANK, On the uniqueness of ground states of non-local equations, Journées Équations aux Dérivées Partielles 2011, Exp. No. V.

[13] R.L. FRANK AND E. LENZMANN, Uniqueness and nondegeneracy of ground states for $(-\Delta)^{s} Q+$ $Q-Q^{\alpha+1}=0$ in $\mathbb{R}$, To appear in Annals of Mathematics.

[14] R.L. Frank, E. Lenzmann And L. Silvestre, Uniqueness of radial solutions for the fractional Laplacian, Preprint, 2013, http //arxiv.org/abs/1302.2652.

[15] E. LenZmann, Uniqueness of ground states for pseudorelativistic Hartree equations, Analysis and PDE 2, No.1, 2009.

[16] Y.Y. LI, On a singularly perturbed elliptic equation, Adv. Differential Equations, 2, No.6 (1997), 955-980. 
[17] E.H. LiEB, H.-T. YAOU, The Chandrasekhar theory of stellar collapse as the limit of quantum mechanics, Comm. Math. Phys., 112 (1987).

[18] D. Mugnai, The pseudorelativistic Hartree equation with a general nonlinearity: existence, non existence and variational identities, Preprint 2012.

[19] S. SECCHI, Ground state solutions for nonlinear fractional Schrödinger equations in $\mathbb{R}^{n}$, J. Math. Phys., in press.

[20] J. TAN, Y. WANG AND J. YANG, Nonlinear fractional field equations, Nonlinear analysis, 72 (2012), 2098-2110. 